\title{
Polarization of Brillouin scattered light in silica nanofibers
}

\author{
Camille Buret, Martin Hauden, Jacques Chrétien, Adrien Godet, Thibaut Sylvestre, Jean-Charles \\ Beugnot, Kien Phan Huy * \\ Institut FEMTO-ST, UMR CNRS 6174, Univ. Bourgogne Franche-Comté, Besançon, France
}

\begin{abstract}
We investigate both theoretically and experimentally the polarization properties of Brillouin light scattering in silica optical nanofibers. Our results show that while all hybrid acoustic waves scatter light without altering the state of polarization, one of the surface acoustic wave generates a depolarized Stokes light. Because of the slight ellipticity of the nanofiber, the surface wave is actually split into two torso-radial modes which give rise to polarization scrambling of the backward Brillouin Stokes signal. Our model also predicts that the polarization of the scattered light can be restored for one specific pump polarization.
\end{abstract}

Keywords: Backward Brillouin scattering, Polarization, silica nanofiber, acoustic modes, ellipticity

\section{INTRODUCTION}

Silica optical nanofibers (ONF) are very attractive nanophotonic platforms for many applications such as optical sensing and nonlinear photonics due to both their wide evanescent field and the tight field confinement in the waist region [1-4]. It has been further shown that ONFs possess unique elastic properties owing to the strong coupling between shear and longitudinal acoustic waves [5]. Among them we find surface acoustic waves (SAW) and hybrid acoustic waves (HAW). Those waves feature both transverse and longitudinal displacements and complex dispersion curves [5]. The Brillouin scattering (BS) in ONFs therefore shows a multi-frequency spectrum in the range 5-10 GHz that is fundamentally different from that of standard optical fibers. These elastic properties have recently been exploited to precise ONF diameter measurements, full tensor elastic nonlinearity measurements, and Brillouin self-cancellation [5-8].

However, polarization properties of Brillouin scattering in optical nanofibers have not been reported yet. They have been only studied so far in standard polarization-maintaining optical fibers and in photonic crystal fibers (PCF) [9-12]. More specifically, both polarized (P) and depolarized (DP) forward guided acoustic wave Brillouin scattering (GAWBS) have been observed in optical fibers. In the former case $(\mathrm{P})$, the elasto-optic interaction generates a pure phase modulation due to thermally-excited radial $\left(\mathrm{R}_{0 \mathrm{~m}}\right)$ acoustic modes. The latter depolarized (DP) case originates from the interaction of light with a degenerated torso-radial $\left(\mathrm{TR}_{2 \mathrm{~m}}\right)$ acoustic mode and to depolarized scattering. The acoustic wavevector matches the wave vectors difference between the two cross-polarized optical modes supported by the birefringent fibers [9-10]. Since backward Brillouin scattering in ONFs features both longitudinal $\mathrm{L}_{0 \mathrm{~m}}$ and torso-radial $\mathrm{TR}_{21}$ modes, we may expect similar effects in a slightly birefringent nanofiber [4].

In this work, we report a complete experimental and theoretical investigation of the polarization properties of the backward Brillouin scattering in tapered optical fibers. We demonstrate that all hybrid acoustic waves (HAWs) scatter light without changing the polarization state, while one of the surface acoustic wave (SAW) generates an unpolarized Stokes light. In the first section, we investigate theoretically the Brillouin scattering in nanofibers and particularly the polarized of depolarized backward Brillouin scattering where SAW and HAW modes are involved. Then we present the experimental setup and the polarization sensitive Brillouin spectrum measurements. Finally, the experimental results are presented and discussed.

\section{THEORETICAL BACKGROUND}

Brillouin backscattering arises from the interaction between two counter-propagating and frequency detuned optical waves and a longitudinal elastic wave. This inelastic scattering is illustrated in Fig. 1 where the pump is depicted by a red arrow, the Stokes scattered light by a blue arrow and the acoustic wave by a green arrow. The standard model used for this light- 
sound interaction is based on three coupled-amplitude equations for the pump, Stokes and the elastic wave [12-14]. In standard single-mode fibers, the acoustic wave can be assumed as a plane wave.

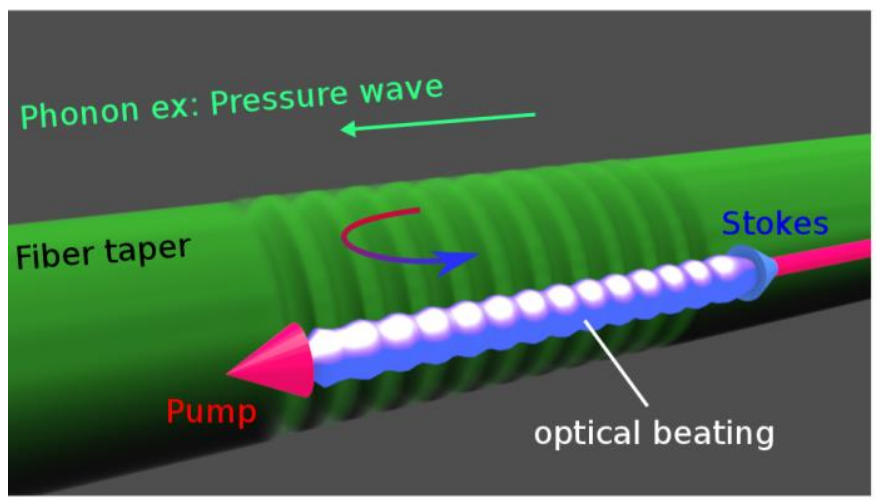

Figure 1. Principle of Brillouin backscattering in an optical fiber taper

However, this assumption is not valid in ONFs as they have no solid cladding and their core size is very close to both the optical and the acoustical wavelengths. This leads to a strong coupling between longitudinal and shear elastic components at the waveguide boundaries. Therefore, the longitudinal elastic wave behaves as an acoustic mode including both longitudinal and shear elastic components. These new hybrid acoustic modes can be split in two groups: L0m that keep a strong longitudinal component and TR2m the torso-radial modes. Their dispersion $\beta_{a}(\Omega)$ can be found by solving the elasto-dynamic equation including the electrostrictive stress [4,15], where $\beta_{a}$ is the wavevector of a given acoustic mode and $\Omega$ its pulsation.

The three coupled-amplitude equations show that efficient scattering occurs when the phase matching condition is satisfied $\beta_{a}(\Omega)=2 \beta_{H E 11}(\omega)$, where $\beta_{H E 11}$ is the wavevector of the fundamental optical mode supported by the nanofiber and $\omega$ is the pump optical pulsation [12-14]. Once the $\beta_{H E 11}(\omega)$ is computed by solving the dispersion equation [12], we can write the phase-matching condition and compute the Brillouin frequency $\Omega$ as a function of the ONF diameter. The result is shown in Figure 2 (Left) where the 15 first Brillouin resonances are shown. For a $1 \mu \mathrm{m}$ diameter ONF, the two lowest resonances between 5 and $6 \mathrm{GHz}$ depicted in blue and green are respectively the TR21 and L01 modes.
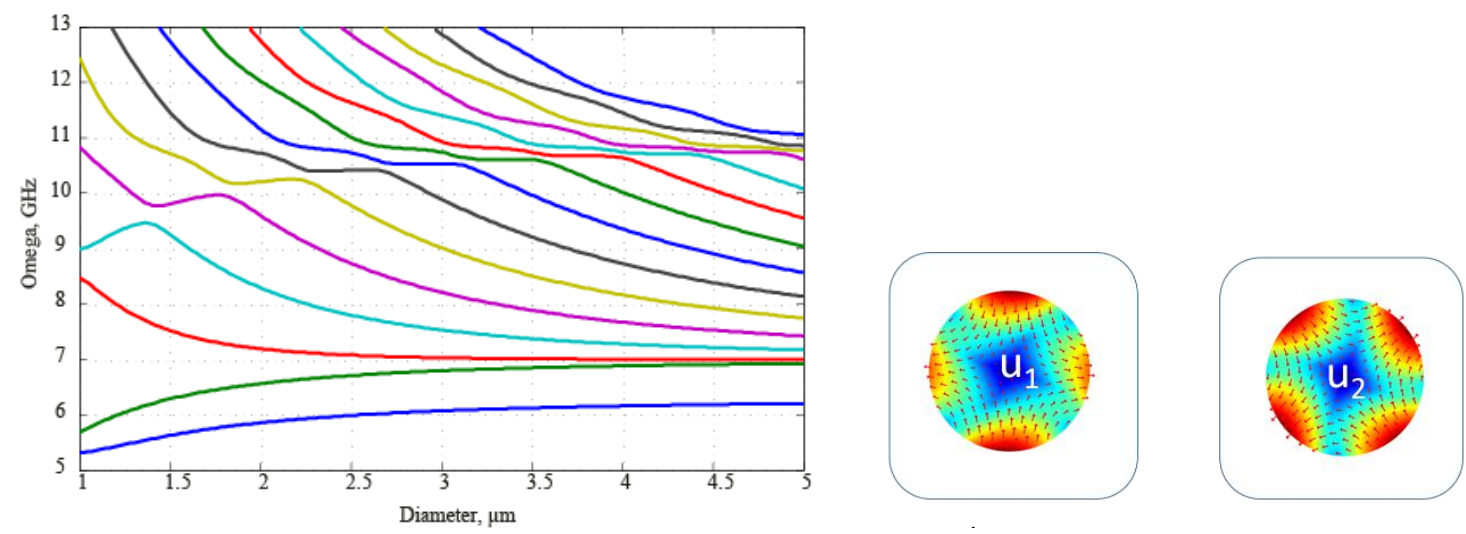

Figure 2. Left: Frequencies of the acoustic modes as a function of the nanofiber diameter resulting from the phase matching condition that takes into account both acoustic and optical mode dispersion, blue line is related to the degenerated TR21 mode. Right: Doubly degenerated TR21 mode transverse displacement (arrow) and kinetic energy (false color) due to slight ellipticity of the nanofiber.

The birefringence of ONFs has been previously measured and studied [16]. As for polarization maintaining and PCF fibers [9-11], the TR21 is then twice degenerate. In order to model the elasto-optic interaction, we solved the dynamic equation including the electrostrictive stress using a finite-element method [15]. We found that two waveforms of twice degenerate TR2 1 acoustic mode $\mathrm{u}_{1}$ and $\mathrm{u}_{2}$, as illustrated in Figure 2 (right). The first mode $\mathrm{u}_{1}$ induces compression along the horizontal 
axis and expansion along the vertical axis without altering the polarization state of the scattered light. However, $\mathrm{u}_{2}$ compression-expansion axes are oriented at $45^{\circ}$ from the horizontal axis, and thus it scatters a vertical polarized light into a horizontal polarized light. Since the scattering of any linear polarized pump will result from the contributions of both twice degenerated TR21 modes [9-10] which are phase shifted randomly because of their thermal excitation, the scattered light polarization is scrambled. The backward Stokes light is thus depolarized.

\section{EXPERIMENTS}

\subsection{Nanofiber fabrication}

The heat brush technique was used to draw nanofibers from standard telecommunication single-mode fibers (SMF-28) [17-18]. The fiber was fixed on two translation stages and softened on its central part with a small butane flame. The flame was regulated using a mass-flow controller and it is kept motionless while the two translation stages stretch the fiber as shown in Figure 3a. The ONF diameter is reduced to the desired shape by monioring the trajectories of the two translation stages.

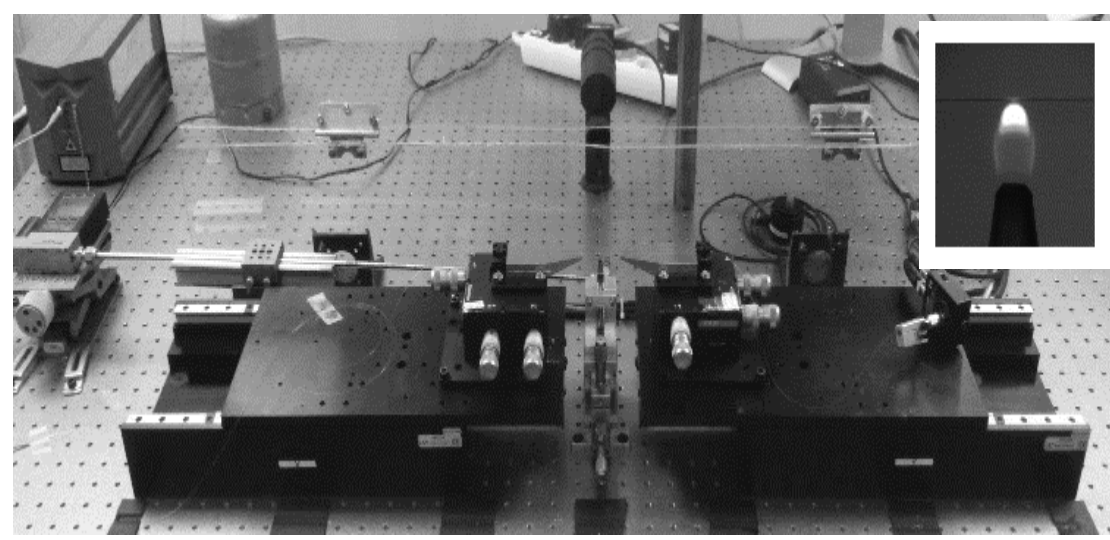

Figure 3. Picture of the pulling bench used for fabrication of silica nanofibers. The Inset shows a zoom on the flame and on the fiber before tapering.

The quality of the tapering process was controlled by measuring the optical transmission [18, 19]. When pulling the fiber, the output transmission is strongly modulated due to optical mode beating and to the excitation of higher-order modes. This beating is due to the transition between a core/clad guidance to a clad/air guidance. As the process goes on, the core vanishes and the light is guided by a large cladding waveguide, the fiber then becomes multimode. As the pulling progresses, the higher-order modes cut-offs are reached and the interferences disappear making the transmission stable. The ONF is thus single-mode. The average transmitted power at the end of the pulling is around $75 \%$ or $1.2 \mathrm{~dB}$ of total loss. Using this pulling technique we were able to draw long and uniform silica nanofibers with diameter as small as 500 $\mathrm{nm}$ and length as long as $40 \mathrm{~mm}$.

\subsection{Polarization-sensitive heterodyne detection setup}

The experimental setup to measure the Brillouin spectrum is shown in Figure 4 and the tapered optical fiber is schematically depicted by a dashed line. A continuous-wave (CW) laser at $\lambda=1550 \mathrm{~nm}$ was first split into two beams using a fiber coupler. One beam was used as pump light and the other one as reference light. The pump light was then amplified by an erbium-doped fiber amplifier (EDFA) up to $33 \mathrm{dBm}$ and injected into the ONF under test through an optical circulator. The backscattered Brillouin signal from the wire was mixed with the reference light, giving rise to an optical beat signal which was further detected with a fast photodiode and analyzed with an electrical spectrum analyzer (ESA). Polarization sensitive measurements were performed using a polarimeter and adding two polarization controllers PC1 and PC2 on both the pump and reference arms. Fiber couplers are also used to measure the polarization state in each arm. Since polarization can be affected by strained fibers, all the fibers are maintained straight. However, we cannot measure the polarization everywhere in the system. Our polarization measurements are thus relative. If the two waves that interfere are in the same 
polarization state, the interference visibility will be maximum and the measured spectrum will show the highest dynamics. If the two polarizations are orthogonal, then the optical beat visibility is null and the spectrum is flat.

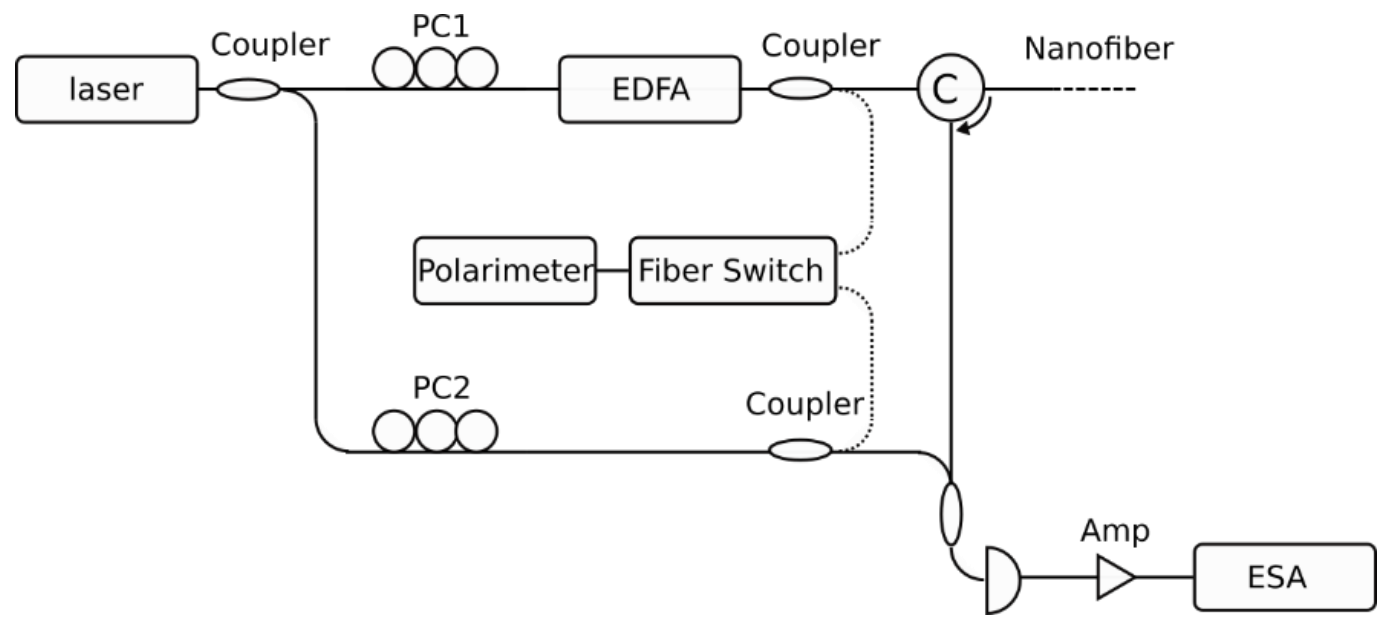

Figure 4. Polarization-sensitive heterodyne detection setup for measuring the backscattering Brillouin spectrum and its polarization properties.

\section{RESULTS AND DISCUSSION}

In the left of Figure 5, we show the backward spontaneous Brillouin scattering spectrum for two specific position of PC2. The blue line corresponds to the maximum visibility of the optical beat giving the most intense SBS spectrum. Above 6 $\mathrm{GHz}$, we find the TR21 and L01 mode at $5.39 \mathrm{GHz}$ and $5.55 \mathrm{GHz}$, respectively. The resonances for frequency above 8 $\mathrm{GHz}$ are $\mathrm{LOm}$ modes with $\mathrm{m}$ superior to 1 . The pedestals seen on each resonance are due to the taper transitions at each end of the ONF [6].
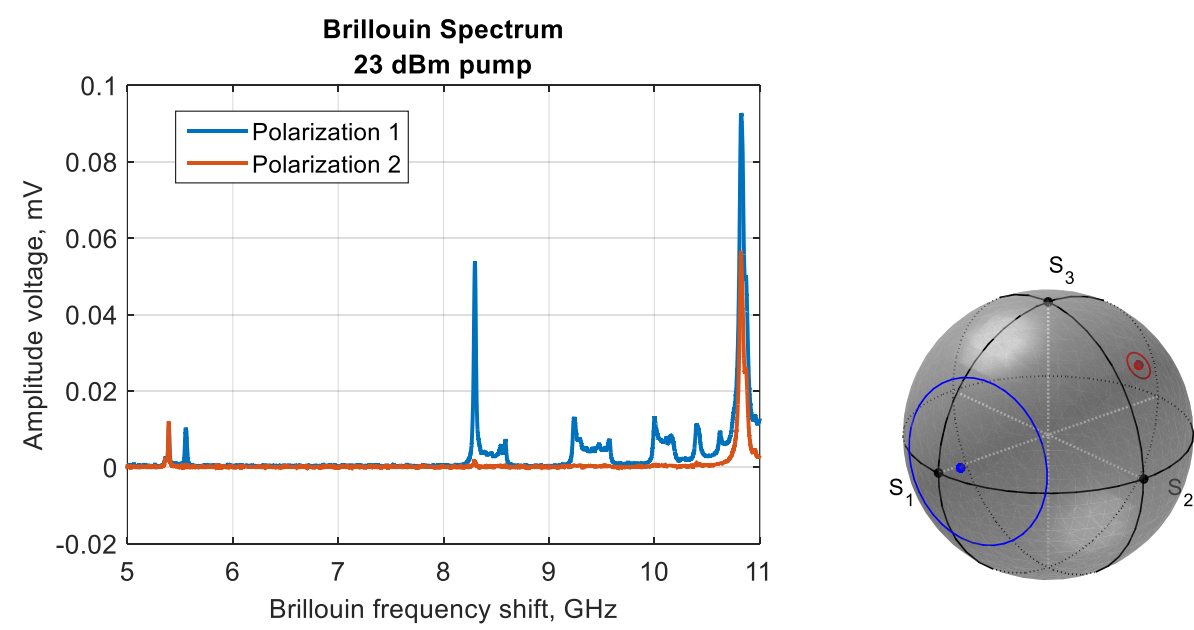

Figure 5. (Left) Spontaneous Brillouin spectrum measurement of the nanofiber for two reference beam polarizations. (Right) Measured polarization of the reference beam on the Poincare Sphere. The TR21 resonance around $5.4 \mathrm{GHz}$ is not affected by the polarization of the reference beam.

The red curve corresponds to the minimum visibility. For both cases, we report the polarization measurement of the reference beam on the Poincaré sphere. One can see they are approximatively orthogonal. The blue and red circle indicates the error bar which is large when measuring constructive interferences due to the slope at the top of the square sine. As for GAWBS measurement in birefringent fibers, the TR21 mode scrambles the polarization of the Brillouin backscattering, as both orthogonal polarization states give the same scattered light amplitude. On the opposite, for the maximum visibility polarization case, all the longitudinal modes LOm behave similarly. It is expected since longitudinal modes do not introduce 
refractive index anisotropy but pure phase modulation [9-10]. This is a very interesting result since GAWBS is a forward scattering that needs to be measured in a Sagnac loop interferometer for increased sensitivity. In such setup, the optical beat results from the interference between two counter-propagating waves in the Sagnac loop. Since the same fiber constitute both arms of the interferometer, it is impossible to tune the reference beam polarization.

In our case, we are able to measure this phenomenon with backward Brillouin scattering in an ONF and to alter both the reference and the pump beam polarizations. Thanks to our finite element resolution of the dynamic equation including the electrostrictive stress, we are able to compare the pump and stokes polarization for the twofold degenerate TR 21 mode. We find that for a circular polarized pump light, Brillouin Stokes polarized light could be obtained from the TR21 resonance. Moreover, the Stokes polarized light would then evolve in an orthogonal state compared to the pump polarization. Using PC1, we experimentally verified this prediction. We adjusted the pump polarization until the TR21 resonance be sensitive to the reference beam polarization. For the previous polarization state 1 , we find for the $5.39 \mathrm{GHz}$ and $5.55 \mathrm{GHz}$ resonances $0.005 \mathrm{mV}$ and $0.025 \mathrm{mV}$ respectively. It is very different from Figure 5 , where the $5.39 \mathrm{GHz}$ resonance is on resonance with the $5.55 \mathrm{GHz}$. For Polarization 2, we measure $0.046 \mathrm{mV}$ and $0.0004 \mathrm{mV}$ for the $5.39 \mathrm{GHz}$ and $5.55 \mathrm{GHz}$ resonances, respectively. It means that both TR21 and L01 modes are now polarization sensitive with orthogonal polarization. To further confirm our theoretical prediction, we compare the polarization state of the pump between this setup and the previous one. We find that both polarizations are separated by a quarter rotation on the Poincaré sphere which is what was predicted by the FEM simulations. We thus demonstrated that, unlike for GAWBS, in backward BS the polarization sensitivity can be restored.

\section{CONCLUSION}

Our results have shown that one of the surface acoustic waves scrambles the input state of polarization, while all other acoustic modes have no effect on the polarization. We also used a fully-realistic theoretical model that confirms our observations. It was shown that, because of the slight ellipticity of the nanofiber, one of the surface acoustic wave is split into doubly degenerate torso-radial modes, giving rise to the depolarization of the Backward Brillouin Stokes optical wave. Our theoretical model also predicts that, for one specific pump configuration, the polarization of the scattered Stokes light can be restored. Our experimental results validate this prediction. This is the first time to our knowledge that this polarization restoration is achieved. Previous GAWBS and forward Raman like measurements in standard and PCF fibers only reported depolarization from the torso-radial modes. This paves the way for future polarization-sensitive applications in nanophotonics or in optomechanics.

\section{AKNOWLEDGEMENT}

This work was supported by the French National Research Agency under Grant Agreement ANR-16-CE24-0010-03, and ANR-15-IDEX-0003 and the EIPHI Graduate school (contract "ANR-17-EURE-0002").

\section{REFERENCES}

[1] Tong, L., Gattass, R. R., Ashcom, J. B., He, S., Lou, J., Shen, M., Maxwell, I.., Mazur, E., "Subwavelengthdiameter silica wires for low-loss optical wave guiding," Nature 426(6968), 816-819 (2003).

[2] Brambilla, G., Xu, F., Horak, P., Jung, Y., Koizumi, F., Sessions, N. P., Koukharenko, E., Feng, X., Murugan, G. S., et al., "Optical fiber nanowires and microwires: fabrication and applications," Adv. Opt. Photon., AOP 1(1), 107-161 (2009).

[3] Belal, M., Song, Z., Jung, Y., Brambilla, G.., Newson, T. P., “Optical fiber microwire current sensor,” Opt. Lett., OL 35(18), 3045-3047 (2010).

[4] Fanjoux, G., Chrétien, J., Godet, A., Phan-Huy, K., Beugnot, J.-C. and Sylvestre, T., "Demonstration of the evanescent Kerr effect in optical nanofibers," Opt. Express, OE 27(20), 29460-29470 (2019).

[5] Beugnot, J.-C., Lebrun, S., Pauliat, G., Maillotte, H., Laude, V. and Sylvestre, T., "Brillouin light scattering from surface acoustic waves in a subwavelength-diameter optical fibre," Nature Communications 5, 5242 (2014). 
[6] Florez, O., Jarschel, P. F., Espinel, Y. a. V., Cordeiro, C. M. B., Alegre, T. P. M., Wiederhecker, G. S.., Dainese, P., "Brillouin scattering self-cancellation," Nature Communications 7, 11759 (2016).

[7] Godet, A., Ndao, A., Sylvestre, T., Pêcheur, V., Lebrun, S., Pauliat, G., Beugnot, J.-C. and Phan Huy K., "Brillouin spectroscopy of optical microfibers and nanofibers," Optica, OPTICA 4(10), 1232-1238 (2017).

[8] Godet, A., Sylvestre, T., Pêcheur, V., Chrétien, J., Beugnot, J.-C. and Phan Huy, K., "Nonlinear elasticity of silica nanofiber," APL Photonics 4(8), 080804 (2019).

[9] Nishizawa, N., Kume, S., Mori, M., Goto, T. and Miyauchi, A., "Experimental analysis of guided acoustic wave Brillouin scattering in PANDA fibers," J. Opt. Soc. Am. B, 12(9), 1651-1655 (1995).

[10] Nishizawa, N., Kume, S., Mori, M., Goto, T. and Miyauchi, A., "Characteristics of Guided Acoustic Wave Brillouin Scattering in Polarization Maintaining Fibers," Optical Review 3(1), 29-33 (1996).

[11] McElhenny, J. E., Pattnaik, R. K. and Toulouse, J., "Dependence of Frequency Shift of Depolarized Guided Acoustic Wave Brillouin Scattering in Photonic Crystal Fibers," J. Lightwave Technol., JLT 29(2), 200-208 (2011).

[12] Beugnot J.-C., Sylvestre T., Maillotte H., Mélin G., and Laude V., "Guided acoustic wave Brillouin scattering in photonic crystal fibers," Opt. Lett. 32, 17-19 (2007).

[13] G. P. Agrawal, Nonlinear Fiber Optics, Academic Press, fifth edition (2012).

[14] R. W. Boyd, Nonlinear Optics, Academic Press, (2008).

[15] Kobyakov, A., Sauer, M.., Chowdhury, D., "Stimulated Brillouin scattering in optical fibers," Adv. Opt. Photon., AOP 2(1), 1-59 (2010).

[16] Beugnot, J.-C. and Laude, V., "Electrostriction and guidance of acoustic phonons in optical fibers," Phys. Rev. B 86(22), 224304 (2012).

[17]Lei, F., Tkachenko, G., Ward, J. M. and Nic Chormaic, S., "Complete Polarization Control for a Nanofiber Waveguide Using Directional Coupling,” Phys. Rev. Applied 11(6), 064041 (2019).

[18][1] Birks, T. A. and Li, Y. W., "The shape of fiber tapers,” Journal of Lightwave Technology 10(4), 432-438 (1992).

[19] Hoffman, J. E., Ravets, S., Grover, J. A., Solano, P., Kordell, P. R., Wong-Campos, J. D., Orozco, L. A.., Rolston, S. L., "Ultrahigh transmission optical nanofibers," AIP Advances 4(6), 067124 (2014).

[20] Ravets, S., Hoffman, J. E., Kordell, P. R., Wong-Campos, J. D., Rolston, S. L.., Orozco, L. A., “Intermodal energy transfer in a tapered optical fiber: optimizing transmission,” J. Opt. Soc. Am. A, JOSAA 30(11), 2361-2371 (2013). 